\title{
NUMERICAL
} METHODS

\section{A Criterion for Coefficient Stability}

\author{
S. V. Lemeshevskii, P. P. Matus, and A. R. Naumovich \\ Belarus State University, Minsk, Belarus \\ Institute of Mathematics, National Academy of Sciences, Minsk, Belarus
}

Received February 6, 2004

\section{INTRODUCTION}

In the analysis of initial-boundary value problems for nonstationary equations of mathematical physics, attention is paid mainly to the stability of the solution with respect to the initial data and the right-hand side. It was proved that the stability of a two-level operator-difference scheme with respect to the initial data is necessary and sufficient for its stability with respect to the right-hand side [1, pp. 95-97].

However, when solving a differential problem, one can face a situation in which the coefficients of the equation are known approximately rather than exactly. (For example, they are obtained with the use of some numerical algorithm, as a result of physical measurements, etc.) It is therefore important to analyze the stability of the solution of the differential problem under perturbations of the initial conditions, the right-hand side, and the operator coefficients (strong stability). Similar problems arise for finite-difference approximations to the differential problem.

The first results concerning the strong stability analysis of operator-difference schemes approximating nonstationary problems of mathematical physics were given in $[2,3]$.

In 1999, Gulin conjectured that stability with respect to the initial data should imply not only stability with respect to the right-hand side but also coefficient stability; this assumption was proved in [4]. Later, Makarov put forward the conjecture that the three notions (stability with respect to the initial data, stability with respect to the right-hand side, and coefficient stability) are equivalent.

In Section 1 of the present paper, by analogy with [5], we give the definition of strong stability of a two-level operator-difference scheme. In Section 2, we introduce the notions of stability of a scheme with respect to the initial data, stability with respect to the right-hand side, and coefficient stability. If only the initial data and the right-hand side are perturbed in the original scheme, then stability with respect to the right-hand side and the initial data, together with the triangle inequality, implies the stability of the difference scheme. However, if the operator coefficients are also perturbed, then the perturbation problem becomes nonlinear and stability with respect to the initial data and the right-hand side and coefficient stability do not imply the strong stability of the difference scheme. In Section 3, we prove the above-mentioned Gulin-Makarov conjecture.

\section{STRONG STABILITY OF TWO-LEVEL OPERATOR-DIFFERENCE SCHEMES}

Let $H_{h}$ be a real finite-dimensional space whose dimension depends on $h$ and can tend to infinity as $|h| \rightarrow 0$. Here $h$ is a vector parameter equipped with a norm $|h|>0$.

Let $\hat{\bar{\omega}}_{\tau}=\left\{t_{n}=t_{n-1}+\tau_{n}, n=0, \ldots, n_{0} ; t_{0}=0, t_{n_{0}}=T\right\}=\hat{\omega}_{\tau} \cup\{T\}$ be an arbitrary grid on the interval $0 \leq t \leq T$ with increments $\tau_{n}=t_{n}-t_{n-1}$.

Consider the Cauchy problem for the two-level operator-difference scheme

$$
B y_{t}+A y=\varphi, \quad t \in \hat{\omega}_{\tau}, \quad y(0)=u_{0},
$$

where $A=A_{h \tau}\left(t_{n}\right)$ and $B=B_{h \tau}\left(t_{n}\right): H_{h} \rightarrow H_{h}$ are linear operators, in general, depending on $\tau$ and $t_{n}, y=y_{n}=y\left(t_{n}\right) \in H_{h}$ is the unknown function, and $\varphi=\varphi_{n}=\varphi\left(t_{n}\right)$ and $u_{0} \in H_{h}$ are given. The operators $A_{h \tau}\left(t_{n}\right)$ and $B_{h \tau}\left(t_{n}\right)$ are bounded for any given $h$ and $\tau$ but, in general, not uniformly bounded with respect to $h$ and $\tau$. 
We use the index-free notation of the theory of difference schemes $[1,6]$ :

$$
y=y_{n}=y\left(t_{n}\right), \quad \hat{y}=y_{n+1}=y\left(t_{n+1}\right), \quad y_{t}=y_{t, n}=\left(y_{n+1}-y_{n}\right) / \tau_{n+1} .
$$

Along with problem (1.1), we consider the perturbed problem

$$
\tilde{B} \tilde{y}_{t}+\tilde{A} \tilde{y}=\tilde{\varphi}, \quad t \in \hat{\omega}_{\tau}, \quad \tilde{y}(0)=\tilde{u}_{0} .
$$

Let us proceed to the study of the strong stability of difference schemes. A solution of the difference Cauchy problem (1.1) is an abstract function $y_{h \tau}\left(t_{n}\right)$ depending on the discrete argument $t_{n} \in \hat{\omega}_{\tau}$ and ranging in $H_{h}$. The input data of the problem consists of the initial vector $y_{0}=y_{0, h \tau} \in H_{h}$ and the right-hand side $\varphi=\varphi_{h \tau}\left(t_{n}\right)$, which is a given abstract function of the discrete argument $t_{n} \in \hat{\omega}_{\tau}$ and ranges in $H_{h}$. Suppose that $H_{h}$ is a normed space, or, more precisely, is equipped with some norms $\left\|y_{n}\right\|_{\left(1_{n}\right)}$ and $\left\|\varphi_{n}\right\|_{\left(2_{n}\right)}$ in which we estimate the perturbation of the solution and the right-hand side of Eq. (1.1), respectively. These norms can depend on $t=t_{n}, h$, and $\left\{\tau_{k}\right\}$.

We introduce the perturbation $\delta=\tilde{y}-y$ of the solution of problem (1.1) with respect to the solution of problem (1.2); it satisfies the problem

$$
\tilde{B} \delta_{t}+\tilde{A} \delta=(\tilde{\varphi}-\varphi)+(B-\tilde{B}) y_{t}+(A-\tilde{A}) y, \quad t \in \hat{\omega}_{\tau}, \quad \delta_{0}=\tilde{u}_{0}-u_{0} .
$$

If the operators $A_{n}$ and $\tilde{A}_{n}$ are treated as mappings $A_{n}, \tilde{A}_{n}: H_{h}^{\left(1_{n}\right)} \rightarrow H_{h}^{\left(2_{n}\right)}$ and the operators $B_{n}$ and $\tilde{B}_{n}$ as mappings $B_{n}, \tilde{B}_{n}: H_{h}^{\left(3_{n}\right)} \rightarrow H_{h}^{\left(4_{n}\right)}$, where $H_{h}^{(\alpha)} \subset H_{h}\left(\alpha=1_{n}, 3_{n}\right)$ and $H_{h} \subset H_{h}^{(\alpha)}$ $\left(\alpha=2_{n}, 4_{n}\right)$, then it is natural to estimate the perturbations of the operator coefficients $A_{n}$ and $B_{n}$ in the operator norms

$$
\begin{aligned}
& \left\|A_{n}-\tilde{A}_{n}\right\|_{\left(3_{n}\right)}=\left\|A_{n}-\tilde{A}_{n}\right\|_{H_{h}^{\left(1_{n}\right)} \rightarrow H_{h}^{\left(2_{n}\right)}}=\sup _{\left\|u_{n}\right\|_{H_{h}^{\left(1_{n}\right)}} \neq 0} \frac{\left\|\left(A_{n}-\tilde{A}_{n}\right) u_{n}\right\|_{H_{h}^{\left(2_{n}\right)}}}{\left\|u_{n}\right\|_{H_{h}^{\left(1_{n}\right)}}}, \\
& \left\|B_{n}-\tilde{B}_{n}\right\|_{\left(4_{n}\right)}=\left\|B_{n}-\tilde{B}_{n}\right\|_{H_{h}^{\left(3_{n}\right)} \rightarrow H_{h}^{\left(4_{n}\right)}}=\sup _{\left\|u_{n}\right\|_{H_{h}^{\left(3_{n}\right)}} \neq 0} \frac{\left\|\left(B_{n}-\tilde{B}_{n}\right) u_{n}\right\|_{H_{h}^{\left(4_{n}\right)}}}{\left\|\tilde{u}_{n}\right\|_{H_{h}^{\left(3_{n}\right)}}} .
\end{aligned}
$$

Following [5], we introduce the notion of strong stability.

Definition 1.1. The operator-difference scheme (1.1) is said to be strongly stable if it is stable under perturbations of the input data, viz., the initial conditions, the right-hand side, and the operator coefficients. In other words, there exist positive constants $M_{k}, k=1,2,3,4$, such that the a priori estimate

$$
\begin{aligned}
\left\|\tilde{y}_{n}-y_{n}\right\|_{\left(1_{n}\right)} \leq & M_{1}\left\|\tilde{u}_{0}-u_{0}\right\|_{\left(1_{0}\right)}+M_{2} \sum_{k=0}^{n-1} \tau_{k+1}\left\|\tilde{\varphi}_{k}-\varphi_{k}\right\|_{\left(2_{k}\right)} \\
& +M_{3} \sum_{k=0}^{n-1} \tau_{k+1}\left\|A_{k}-\tilde{A}_{k}\right\|_{\left(3_{k}\right)}+M_{4} \sum_{k=0}^{n-1} \tau_{k+1}\left\|B_{k}-\tilde{B}_{k}\right\|_{\left(4_{k}\right)}
\end{aligned}
$$

is valid.

Here and throughout the following, we assume that $0 \leq n \leq n_{0}<\infty$.

Example 1.1. The main problem in the derivation of estimates of the form (1.4) is to choose the spaces in which the operators $A_{n}, \tilde{A}_{n}$ and $B_{n}, \tilde{B}_{n}$ act so as to ensure that the norms of perturbations of the operator coefficients are bounded. 
By way of example, we consider the constant operator $A y=-\left(a y_{\bar{x}}\right)_{x}, x \in \omega_{h}, y_{0}=y_{N}=0$, where $\omega_{h}$ is the uniform grid with increment $h=1 / N$ on $[0,1]$ and $a \neq a(t) \geq \delta>0$ and $y=y(t)$ are grid functions. Then $\tilde{A} y=-\left(\tilde{a} y_{\bar{x}}\right)_{x}, x \in \omega_{h}, y_{0}=y_{N}=0, \tilde{a} \neq \tilde{a}(t) \geq \delta>0$, and $(A-\tilde{A}) y=-\left((a-\tilde{a}) y_{\bar{x}}\right)_{x}, x \in \omega_{h}, y_{0}=y_{N}=0$. The space $H_{h}$ is defined as the set of grid functions $y_{i}=y\left(x_{i}\right)$ defined on $\omega_{h}$ and vanishing for $i=0, N$. The inner product and the norm in $H_{h}$ are given by the formulas

$$
(y, v)=\sum_{i=1}^{N-1} y_{i} v_{i} h, \quad\|y\|=\sqrt{(y, y)} .
$$

As $H_{h}^{\left(1_{n}\right)}$ we take, say, the normed space $H_{\tilde{A}^{*} \tilde{A}}$ with the norm $\|\tilde{A} u\|, u \in H_{h}$, and $H_{h}^{\left(2_{n}\right)}=H_{h}$. If $|\tilde{a}(x)-a(x)| \leq \alpha_{1}<+\infty$ and $\left|\tilde{a}_{x}(x)-a_{x}(x)\right| \leq \alpha_{2}<+\infty$, then

$$
\|(A-\tilde{A}) u\|_{H_{h}} \leq \alpha\|\tilde{A} u\|_{H_{h}}=\alpha\|u\|_{H_{\tilde{A}^{*} \tilde{A}}}
$$

consequently, for the perturbation of the operator $A$, we have

$$
\|A-\tilde{A}\|_{H_{\tilde{A}^{*} \tilde{A}} \rightarrow H_{h}} \leq \alpha<+\infty .
$$

\section{STABILITY WITH RESPECT TO THE INPUT DATA}

The notion of stability with respect to the initial data and the right-hand side, as well as the notion of coefficient stability for a stationary problem, was introduced in [6] for the difference scheme (1.1).

Here we introduce the corresponding notions for the case of a nonstationary problem and perturbations of operator coefficients.

Along with problem (1.2), we consider the problems

$$
\begin{array}{lll}
B \tilde{y}_{t}^{(1)}+A \tilde{y}^{(1)}=\varphi, & t \in \hat{\omega}_{\tau}, & \tilde{y}^{(1)}(0)=\tilde{u}_{0}, \\
B \tilde{y}_{t}^{(2)}+A \tilde{y}^{(2)}=\tilde{\varphi}, & t \in \hat{\omega}_{\tau}, & \tilde{y}^{(2)}(0)=u_{0}, \\
\tilde{B} \tilde{y}_{t}^{(3)}+\tilde{A} \tilde{y}^{(3)}=\varphi, & t \in \hat{\omega}_{\tau}, & \tilde{y}^{(3)}(0)=u_{0} .
\end{array}
$$

Definition 2.1. The operator-difference scheme (1.1) is said to be stable with respect to the initial data if there exists a positive constant $M_{1}$ such that

$$
\left\|\tilde{y}_{n}^{(1)}-y_{n}\right\|_{\left(1_{n}\right)} \leq M_{1}\left\|\tilde{u}_{0}-u_{0}\right\|_{\left(1_{0}\right)} .
$$

Definition 2.2. The operator-difference scheme (1.1) is said to be stable with respect to the right-hand side if there exists a positive constant $M_{2}$ such that

$$
\left\|\tilde{y}_{n}^{(2)}-y_{n}\right\|_{\left(1_{n}\right)} \leq M_{2} \sum_{k=1}^{n-1} \tau_{k+1}\left\|\tilde{\varphi}_{k}-\varphi_{k}\right\|_{\left(2_{k}\right)} .
$$

Definition 2.3. The operator-difference scheme (1.1) is stable with respect to the operator coefficients if there exist positive constants $M_{3}$ and $M_{4}$ such that

$$
\left\|\tilde{y}_{n}^{(3)}-y_{n}\right\|_{\left(1_{n}\right)} \leq M_{3} \sum_{k=1}^{n-1} \tau_{k+1}\left\|\tilde{A}_{k}-A_{k}\right\|_{\left(3_{k}\right)}+M_{4} \sum_{k=1}^{n-1} \tau_{k+1}\left\|\tilde{B}_{k}-B_{k}\right\|_{\left(4_{k}\right)} .
$$


Furthermore, we introduce the perturbations of the solution of problem (1.1) with respect to the solutions of problems (1.2a)-(1.2c): $\delta^{(k)}=\tilde{y}^{(k)}-y, k=1,2,3$. For $\delta^{(k)}(k=1,2,3)$, we obtain the problems

$$
\begin{aligned}
& B \delta_{t}^{(1)}+A \delta^{(1)}=0, \quad t \in \hat{\omega}_{\tau}, \quad \delta_{0}^{(1)}=\tilde{u}_{0}-u_{0}, \\
& B \delta_{t}^{(2)}+A \delta^{(2)}=\tilde{\varphi}-\varphi, \quad t \in \hat{\omega}_{\tau}, \quad \delta_{0}^{(2)}=0, \\
& B \delta_{t}^{(3)}+A \delta^{(3)}=(B-\tilde{B}) \tilde{y}_{t}^{(3)}+(A-\tilde{A}) \tilde{y}^{(3)}, \quad t \in \hat{\omega}_{\tau}, \quad \delta_{0}^{(3)}=0 .
\end{aligned}
$$

Moreover, $\delta^{(3)}$ is a solution of the problem

$$
\tilde{B} \delta_{t}^{(3)}+\tilde{A} \delta^{(3)}=(B-\tilde{B}) y_{t}+(A-\tilde{A}) y, \quad t \in \hat{\omega}_{\tau}, \quad \delta_{0}^{(3)}=0 .
$$

Note that since problem (1.3) is nonlinear in the case of perturbation of the operator coefficients, and since $\delta \neq \delta^{(1)}+\delta^{(2)}+\delta^{(3)}$, it follows that, unlike the case of perturbations of the initial data and the right-hand side alone, it is impossible to use the triangle inequality to obtain a strong stability estimate for $\delta$ from the estimates for $\delta^{(1)}, \delta^{(2)}$, and $\delta^{(3)}$.

We assume that the Cauchy problem (1.1) is solvable, i.e., the inverse operator $B_{n}^{-1}$ exists. Since the operator $B_{n}=B_{h \tau}\left(t_{n}\right)$ is bounded for given $h$ and $\tau_{n}$, i.e.,

$$
\left\|B_{n} u_{n}\right\| \leq m\left\|u_{n}\right\| \quad(m>0)
$$

it follows that the operator $B_{n}^{-1}$ satisfies $\left\|B_{n}^{-1} u_{n}\right\| \geq(1 / m)\left\|u_{n}\right\|$ for given $h$ and $\tau_{n}$; consequently, the expression $\left\|B_{n}^{-1} u_{n}\right\|$ is a norm. The scheme (1.1) can be represented in the form [1]

$$
y_{n+1}=S_{n+1} y_{n}+\tau_{n+1} B_{n}^{-1} \varphi_{n}, \quad n=0,1, \ldots, \quad y_{0} \in H,
$$

where the operator $S_{n+1}$ of transition from level $n$ to level $n+1$ is equal to $S_{n+1}=E-\tau_{n+1} B_{n}^{-1} A_{n}$.

By successively using formula (2.7), we obtain

$$
y_{n}=T_{n, 0} y_{0}+\sum_{k=0}^{n-1} \tau_{k+1} T_{n, k+1} B_{k}^{-1} \varphi_{k} .
$$

Furthermore,

$$
y_{n}=T_{n, k} y_{k}+\sum_{j=k}^{n-1} \tau_{j+1} T_{n, j+1} B_{j}^{-1} \varphi_{j}
$$

for each $k \leq n-1$. Here $T_{n, k}$ is the operator of transition from level $k$ to level $n$ :

$$
T_{n, k}=S_{n} S_{n-1} \cdots S_{k+1}, \quad T_{n, n}=E,
$$

and $T_{n, 0}$ is the resolving operator.

By analogy with [1], we say that the scheme (1.1) is uniformly stable with respect to the initial data if there exists a positive constant $M_{1}$ independent of $h,\left\{\tau_{k}\right\}$, and the choice of the initial data such that

$$
\left\|\tilde{y}_{n}^{(1)}-y_{n}\right\|_{\left(1_{n}\right)} \leq M_{1}\left\|\tilde{y}_{k}^{(1)}-y_{k}\right\|_{\left(1_{k}\right)}, \quad k=0, \ldots, n-1, \quad n=1,2, \ldots
$$

Obviously, the estimate (2.9) is valid if and only if the operator $T_{n, k}$ of transition from level $k$ to level $n$ is bounded uniformly with respect to $n$ and $k$ :

$$
\left\|T_{n, k}\right\| \leq M_{1}, \quad 0 \leq k \leq n-1, \quad n=1,2, \ldots
$$




\section{THE RELATIONSHIP BETWEEN STABILITY WITH RESPECT \\ TO THE INITIAL DATA, STABILITY WITH RESPECT TO \\ THE RIGHT-HAND SIDE, AND COEFFICIENT STABILITY}

Theorem 3.1. Suppose that the operators $B_{n}, \tilde{B}_{n}$, and $\tilde{A}_{n}$ are continuously invertible for all $1 \leq n \leq n_{0}<+\infty$, the operators $\tilde{A}_{n}, \tilde{A}_{k}$, and $\tilde{B}_{k}$ commute for all $0 \leq k, n \leq n_{0}$, the operators $S_{1}$ and $\tilde{S}_{1}$ are bounded in the norm $\|\cdot\|_{H_{h} \rightarrow H_{h}}$, i.e.,

$$
\left\|S_{1}\right\|=\left\|S_{1}\right\|_{H_{h} \rightarrow H_{h}} \leq M, \quad\left\|\tilde{S}_{1}\right\|=\left\|\tilde{S}_{1}\right\|_{H_{h} \rightarrow H_{h}} \leq \tilde{M}
$$

and the operator $\tilde{A}_{n}$ satisfies the inequality

$$
\left\|\left(\tilde{A}_{n+1}-\tilde{A}_{n}\right) u_{n}\right\|_{H_{h}} \leq c_{0} \tau_{n+1}\left\|\tilde{A}_{n} u_{n}\right\|_{H_{h}} .
$$

Then the following assertions are equivalent:

(1) the scheme (1.1) is uniformly stable with respect to the initial data;

(2) the scheme (1.1) is stable with respect to the right-hand side;

(3) the scheme (1.1) is coefficient stable.

Proof. $(1) \Rightarrow(2)$. Suppose that the scheme (1.1) is uniformly stable with respect to the initial data, i.e., an estimate of the form (2.9) is valid for problem (2.4):

$$
\left\|\delta_{n}^{(1)}\right\|_{\left(1_{n}\right)} \leq M_{1}\left\|\delta_{k}^{(1)}\right\|_{\left(1_{k}\right)}, \quad k=0, \ldots, n-1, \quad n=1,2, \ldots
$$

Then inequality (2.10) is also valid with $\left\|T_{n, k}\right\|=\left\|T_{n, k}\right\|_{H_{h} \rightarrow H_{h}}$.

By virtue of (2.8), the solution of problem (2.5) is given by the formula

$$
\delta_{n}^{(2)}=\sum_{k=0}^{n-1} \tau_{k+1} T_{n, k+1} B_{k}^{-1}\left(\tilde{\varphi}_{k}-\varphi_{k}\right) .
$$

This, together with (2.10), implies that

$$
\begin{aligned}
\left\|\delta_{n}^{(2)}\right\|_{\left(1_{n}\right)} & \leq \sum_{k=0}^{n-1} \tau_{k+1}\left\|T_{n, k+1}\right\|\left\|B_{k}^{-1}\left(\tilde{\varphi}_{k}-\varphi_{k}\right)\right\|_{\left(1_{k+1}\right)} \\
& \leq M_{1} \sum_{k=0}^{n-1} \tau_{k+1}\left\|B_{k}^{-1}\left(\tilde{\varphi}_{k}-\varphi_{k}\right)\right\|_{\left(1_{k+1}\right)} \\
& \leq M_{1} \sum_{k=0}^{n-1} \tau_{k+1}\left\|\tilde{\varphi}_{k}-\varphi_{k}\right\|_{\left(2_{k}\right)}
\end{aligned}
$$

Therefore, the scheme (1.1) is stable with respect to the right-hand side under the norm compatibility condition

$$
\left\|v_{k}\right\|_{\left(2_{k}\right)}=\left\|B_{k}^{-1} v_{k}\right\|_{\left(1_{k+1}\right)} .
$$

$(2) \Rightarrow(3)$. Suppose that the scheme (1.1) is stable with respect to the right-hand side, i.e., the estimate (3.5) be valid. We shall prove the boundedness of the operators $T_{n, k}$ by the technique in [1]. We choose the perturbed problem $(1.2 \mathrm{~b})$ so as to ensure that $\tau_{k+1} B_{k}^{-1}\left(\tilde{\varphi}_{k}-\varphi_{k}\right)=\delta_{k, k_{0}} \eta$, where $\delta_{k, k_{0}}$ is the Kronecker delta. Then from (3.5), we have

$$
\left\|\delta_{n}^{(2)}\right\|_{\left(1_{n}\right)} \leq M_{1}\|\eta\|_{\left(1_{k_{0}+1}\right)}, \quad n=1, \ldots, n_{0}, \quad k_{0}=0, \ldots, n-1 .
$$

DIFFERENTIAL EQUATIONS $\quad$ Vol. $40 \quad$ No. $7 \quad 2004$ 
On the other hand, from (3.4), we obtain

$$
\delta_{n}^{(2)}=T_{n, k_{0}+1} \eta, \quad\left\|\delta_{n}^{(2)}\right\|_{\left(1_{n}\right)}=\left\|T_{n, k_{0}+1} \eta\right\|_{\left(1_{n}\right)} \leq M_{1}\|\eta\|_{\left(1_{k_{0}+1}\right)},
$$

and consequently, $\left\|T_{n, k}\right\| \leq M_{1}$ for all $k=1, \ldots, n-1$. Since $T_{n, 0}=T_{n, 1} S_{1}$, it follows from (3.1) that $\left\|T_{n, 0}\right\| \leq\left\|T_{n, 1}\right\|\left\|S_{1}\right\| \leq M_{1} M$, i.e., the norms of $T_{n, k}$ are bounded for all $k=0, \ldots, n$ and all $n=1, \ldots, n_{0}$.

Let us estimate the norms of the operators $\tilde{T}_{n, k}$ and $\tilde{S}_{k}=E+\tau_{k+1} \tilde{B}_{k}^{-1} \tilde{A}_{k}^{-1}$. To this end, we consider the quantity $\nu_{k}=\delta_{k}^{(1)}+\delta_{k}^{(2)}+\delta_{k}^{(3)}$. One can readily see that $\nu$ is a solution of the problem

$$
\tilde{B} \nu_{t}+\tilde{A} \nu=\tilde{B}\left(\delta^{(1)}+\delta^{(2)}-y\right)_{t}+\tilde{A}\left(\delta^{(1)}+\delta^{(2)}-y\right)+\varphi, \quad t \in \hat{\omega}_{\tau}, \quad \nu_{0}=\tilde{u}_{0}-u_{0}=\delta_{0},
$$

and the representation

$$
\nu_{n}=\tilde{T}_{n} \delta_{0}+\sum_{k=0}^{n-1} \tau_{k+1} \tilde{T}_{n, k+1} \tilde{B}_{k}^{-1}\left(\tilde{B}_{k}\left(\delta^{(1)}+\delta^{(2)}-y\right)_{t, k}+\tilde{A}_{k}\left(\delta^{(1)}+\delta^{(2)}-y\right)_{k}+\varphi_{k}\right)
$$

is valid. Since the solution of problem (1.3) can be represented in the form

$$
\delta_{n}=\tilde{T}_{n} \delta_{0}+\sum_{k=0}^{n-1} \tau_{k+1} \tilde{T}_{n, k+1} \tilde{B}_{k}^{-1}\left(\tilde{\varphi}_{k}-\tilde{B}_{k} y_{t, k}-\tilde{A}_{k} y_{k}\right)
$$

we have

$$
\begin{aligned}
\delta_{n}= & \delta_{n}^{(1)}+\delta_{n}^{(2)}+\delta_{n}^{(3)} \\
& +\sum_{k=0}^{n-1} \tau_{k+1} \tilde{T}_{n, k+1} \tilde{B}_{k}^{-1}\left(\tilde{\varphi}_{k}-\varphi_{k}-\tilde{B}_{k}\left(\delta^{(1)}+\delta^{(2)}\right)_{t, k}-\tilde{A}_{k}\left(\delta^{(1)}+\delta^{(2)}\right)_{k}\right) .
\end{aligned}
$$

From the last relation, for $\delta^{(2)}$, we obtain

$$
\begin{aligned}
\delta_{n}^{(2)}= & \left(\tilde{T}_{n}-T_{n}\right) \delta_{0}+\sum_{k=0}^{n-1} \tau_{k+1} \tilde{T}_{n, k+1} \tilde{B}_{k}^{-1} \\
& \times\left(\tilde{B}_{k}\left(\delta^{(1)}+\delta^{(2)}+y\right)_{t, k}+\tilde{A}_{k}\left(\delta^{(1)}+\delta^{(2)}+y\right)_{k}-\tilde{\varphi}_{k}\right) .
\end{aligned}
$$

We choose the initial data $\tilde{u}_{0}$ and $u_{0}$ and the right-hand sides $\tilde{\varphi}_{k}$ and $\varphi_{k}$ as follows:

$$
\begin{aligned}
& \tilde{u}_{0}=u_{0}, \\
& \varphi_{k}=\tilde{B}_{k}\left(\delta^{(1)}+\delta^{(2)}+y\right)_{t, k}+\tilde{A}_{k}\left(\delta^{(1)}+\delta^{(2)}+y\right)_{k}-\frac{1}{\tau_{k+1}} \delta_{k, k_{0}}\left(\tilde{B}_{k}+\tilde{B}_{k}\right) \eta, \\
& \tilde{\varphi}_{k}=\varphi_{k}+\frac{1}{\tau_{k+1}} \delta_{k, k_{0}} B_{k} \eta .
\end{aligned}
$$

Then $\delta_{n}^{(2)}=\tilde{T}_{n, k_{0}+1} \eta, \tau_{k+1} B_{k}^{-1}\left(\tilde{\varphi}_{k}-\varphi_{k}\right)=\delta_{k, k_{0}} \eta$, and it follows from the estimate (3.5) that $\left\|\tilde{T}_{n, k_{0}+1} \eta\right\|_{\left(1_{n}\right)} \leq M_{1}\|\eta\|_{\left(1_{k_{0}+1}\right)}$. Since $k_{0}$ and $n$ are arbitrary, we have the estimate

$$
\left\|\tilde{T}_{n, k}\right\| \leq M_{1} \quad \text { for all } \quad 1 \leq k \leq n, \quad n=1,2, \ldots
$$

The formula $\tilde{T}_{n, 0}=\tilde{T}_{n, 1} \tilde{S}_{1}$ implies the estimate

$$
\left\|\tilde{T}_{n, 0}\right\| \leq\left\|\tilde{T}_{n, 1}\right\|\left\|\tilde{S}_{1}\right\| \leq M_{1} M_{3}
$$

Consequently, the norms of $\tilde{T}_{n, k}$ are bounded for all $0 \leq k<n$ and $n=1, \ldots, n_{0}$. 
By virtue of (2.8), the solution of problem (2.6) is given by the formula

$$
\delta_{n}^{(3)}=\sum_{k=0}^{n-1} \tau_{k+1} T_{n, k+1} B_{k}^{-1}\left(\left(B_{k}-\tilde{B}_{k}\right) \tilde{y}_{t, k}^{(3)}+\left(A_{k}-\tilde{A}_{k}\right) \tilde{y}_{k}^{(3)}\right) .
$$

Hence we have

$$
\begin{aligned}
\left\|\delta_{n}^{(3)}\right\|_{\left(1_{n}\right)} & \leq \sum_{k=0}^{n-1} \tau_{k+1}\left\|T_{n, k+1}\right\|\left(\left\|B_{k}^{-1}\left(B_{k}-\tilde{B}_{k}\right) \tilde{y}_{t, k}^{(3)}\right\|_{\left(1_{k}\right)}+\left\|B_{k}^{-1}\left(A_{k}-\tilde{A}_{k}\right) \tilde{y}_{k}^{(3)}\right\|_{\left(1_{k}\right)}\right) \\
& \leq M_{1} \sum_{k=0}^{n-1} \tau_{k+1}\left(\left\|A_{k}-\tilde{A}_{k}\right\|_{\left(3_{k}\right)}\left\|\tilde{A}_{k} \tilde{y}_{k}^{(3)}\right\|_{\left(1_{k}\right)}+\left\|B_{k}-\tilde{B}_{k}\right\|_{\left(4_{k}\right)}\left\|\tilde{B}_{k} \tilde{y}_{t, k}^{(3)}\right\|_{\left(1_{k}\right)}\right),
\end{aligned}
$$

where

$$
\begin{aligned}
& \left\|A_{k}-\tilde{A}_{k}\right\|_{\left(3_{k}\right)}=\left\|A_{k}-\tilde{A}_{k}\right\|_{H_{\tilde{A}_{k}^{*} \tilde{A}_{k}} \rightarrow H_{B_{k}^{*-1} B_{k}^{-1}}} \\
& =\sup _{\left\|\tilde{A}_{k} u_{k}\right\|_{\left(1_{k}\right)} \neq 0}\left(\left\|B_{k}^{-1}\left(A_{k}-\tilde{A}_{k}\right) u_{k}\right\|_{\left(1_{k}\right)} /\left\|\tilde{A}_{k} u_{k}\right\|_{\left(1_{k}\right)}\right), \\
& \left\|B_{k}-\tilde{B}_{k}\right\|_{\left(4_{k}\right)}=\left\|B_{k}-\tilde{B}_{k}\right\|_{H_{\tilde{B}_{k}^{*} \tilde{B}_{k}} \rightarrow H_{B_{k}^{*-1} B_{k}^{-1}}} \\
& =\sup _{\left\|\tilde{B}_{k} u_{k}\right\|_{\left(1_{k}\right)} \neq 0}\left(\left\|B_{k}^{-1}\left(B_{k}-\tilde{B}_{k}\right) u_{k}\right\|_{\left(1_{k}\right)} /\left\|\tilde{B}_{k} u_{k}\right\|_{\left(1_{k}\right)}\right) .
\end{aligned}
$$

From Eq. (1.2c), we obtain $\left\|\tilde{B}_{k} \tilde{y}_{t, k}\right\|_{\left(1_{k}\right)} \leq\left\|\varphi_{k}\right\|_{\left(1_{k}\right)}+\left\|\tilde{A}_{k} \tilde{y}_{k}^{(3)}\right\|_{\left(1_{k}\right)}$.

Let us estimate $\left\|\tilde{A}_{k} \tilde{y}_{k}^{(3)}\right\|_{\left(1_{k}\right)}$. The solution of problem (1.2c) is given by the formula

$$
\tilde{y}_{n}^{(3)}=\tilde{T}_{n} u_{0}+\sum_{k=0}^{n-1} \tau_{k+1} \tilde{T}_{n, k+1} \tilde{B}_{k}^{-1} \varphi_{k}
$$

Then, by taking account of the estimate (3.6), the continuous invertibility of the operator $\tilde{B}_{k}$, and the fact that

$$
\left\|\tilde{A}_{k} v_{k}\right\|_{\left(1_{k}\right)} \leq \varrho\left\|\tilde{A}_{k-1} v_{k}\right\|_{\left(1_{k-1}\right)}, \quad \varrho=\max _{1 \leq k \leq n} \varrho_{k}, \quad \varrho_{k}=1+C_{0} \tau_{k}
$$

which follows from condition (3.2), we obtain the inequality

$$
\left\|\tilde{A}_{k} \tilde{y}_{k}^{(3)}\right\| \leq M_{1}\left(\varrho^{k}\left\|\tilde{A}_{0} u_{0}\right\|_{\left(1_{0}\right)}+\sum_{j=0}^{k-1} \tau_{j+1} \varrho^{k-j}\left\|\tilde{A}_{j} \tilde{B}_{j}^{-1} \varphi_{j}\right\|_{\left(1_{j}\right)}\right) .
$$

With regard to the last estimate, we obtain the inequality

$$
\left\|\delta_{n}^{(3)}\right\|_{\left(1_{n}\right)} \leq M_{3} \sum_{k=0}^{n-1} \tau_{k+1}\left\|A_{k}-\tilde{A}_{k}\right\|_{\left(3_{k}\right)}+M_{4} \sum_{k=0}^{n-1} \tau_{k+1}\left\|B_{k}-\tilde{B}_{k}\right\|_{\left(4_{k}\right)}
$$

where

$$
M_{i} \leq M_{1}\left(\varrho^{n}\left\|\tilde{A}_{0} u_{0}\right\|+(i-3) \max _{0 \leq k \leq n-1}\left\|\varphi_{k}\right\|_{\left(1_{k}\right)}+\sum_{j=0}^{n-1} \tau_{j+1} \varrho^{n-j}\left\|\tilde{A}_{j} \tilde{B}_{j}^{-1} \varphi_{j}\right\|_{\left(1_{j}\right)}\right), \quad i=3,4 .
$$

Therefore, the scheme (1.1) is stable with respect to the operator coefficients.

DIFFERENTIAL EQUATIONS $\quad$ Vol. $40 \quad$ No. 72004 
$(3) \Rightarrow(1)$. Now we suppose that the scheme (1.1) is stable with respect to the operator coefficients, i.e., the estimate (2.3) is valid, where the constants $M_{3}$ and $M_{4}$ satisfy inequalities (3.7).

We choose the perturbed operators $\tilde{B}_{k}$ and $\tilde{A}_{k}$ so as to ensure that $\left(B_{k}-\tilde{B}_{k}\right) u_{k}=0$ and $\left(A_{k}-\tilde{A}_{k}\right) u_{k}=\delta_{k, k_{0}} \tau_{k_{0}+1}^{-1} B_{k} u_{k}$ for all $u_{k} \in H_{h}$ and $k=0, \ldots, n_{0}-1$. Then $\delta_{k}^{(3)}=T_{n, k_{0}+1} \tilde{y}_{k_{0}}^{(3)}$ and

$$
\sum_{k=0}^{n-1} \tau_{k+1}\left\|A_{k}-\tilde{A}_{k}\right\|_{\left(3_{k}\right)}=\sup _{\left\|\tilde{A}_{k_{0}} u_{k_{0}}\right\|_{\left(1_{k_{0}}\right)} \neq 0} \frac{\left\|u_{k_{0}}\right\|_{\left(1_{k_{0}}\right)}}{\left\|\tilde{A}_{k_{0}} u_{k_{0}}\right\|_{\left(1_{k_{0}}\right)}}=\sup _{\left\|v_{k_{0}}\right\|_{\left(1_{k_{0}}\right)} \neq 0} \frac{\left\|\tilde{A}_{k_{0}}^{-1} v_{k_{0}}\right\|_{\left(1_{k_{0}}\right)}}{\left\|v_{k_{0}}\right\|_{\left(1_{k_{0}}\right)}}=\left\|\tilde{A}_{k_{0}}^{-1}\right\| .
$$

From the last relations and the estimate (2.3), we obtain the estimate

$$
\left\|T_{n, k_{0}+1} \tilde{y}_{k_{0}}^{(3)}\right\|_{\left(1_{k_{0}}\right)} \leq M_{3}\left\|\tilde{A}_{k_{0}}^{-1}\right\|
$$

and consequently, the operators $T_{n, k}$ are bounded for all $k=1, \ldots, n-1$, since the operator $\tilde{A}_{k_{0}}$ is continuously invertible and hence $\left\|\tilde{A}_{k_{0}}^{-1}\right\| \leq m, m=$ const $>0$. Condition (3.1) implies that the operators $T_{n, k}$ are also bounded for all $0 \leq k \leq n-1$ and $n=1, \ldots, n_{0}$.

Since the solution of problem (2.4) is given by the formula $\delta_{n}^{(1)}=T_{n, k} \delta_{k}^{(1)}$ for each $k \leq n-1$, we have the estimate $(3.3)$ for $\delta_{n}^{(1)}$, which implies the uniform stability of the scheme (1.1) with respect to the initial data.

Note that, by using the method in [7], one can prove the equivalence of the notions of stability with respect to the initial data, stability with respect to the right-hand side, and coefficient stability without requiring that the operators $\tilde{A}_{n}, \tilde{A}_{k}$, and $\tilde{B}_{k}$ commute.

\section{ACKNOWLEDGMENTS}

The authors are grateful to V.L. Makarov for useful remarks, which made for the improvement of the paper.

\section{REFERENCES}

1. Samarskii, A.A. and Gulin, A.V., Ustoichivost' raznostnykh skhem (Stability of Difference Schemes), Moscow, 1973.

2. Samarskii, A.A., Vabishchevich, P.N., and Matus, P.P., Dokl. RAN, 1997, vol. 356, no. 4, pp. 455-457.

3. Samarskii, A.A., Vabishchevich, P.N., and Matus, P.P., Raznostnye skhemy s operatornymi mnozhitelyami (Difference Schemes with Operator Factors), Moscow, 1998.

4. Samarskii, A.A., Gulin, A.V., and Matus, P.P., Dokl. RAN, 2000, vol. 373, no. 3, pp. 304-306.

5. Matus, P., Comp. Meth. Appl. Math., 2002, vol. 2, no. 1, pp. 50-91.

6. Samarskii, A.A., Teoriya raznostnykh skhem (Theory of Difference Schemes), Moscow, 1977.

7. Gavrilyuk, I.P., Makarov, V.L., and Ryabichev, V.L., Comp. Meth. Appl. Math., 2003, vol. 3, no. 1, pp. 86-115. 